\title{
Antihyperlipidemic Activity of Aloe succotrina in Rats: Possibly Mediated by Inhibition of HMG-CoA Reductase
}

\author{
Dinesh Dhingra, ${ }^{1}$ Deepak Lamba, ${ }^{1}$ Ramesh Kumar, ${ }^{2}$ \\ Pashupati Nath, ${ }^{2}$ and Satyaprakash Gauttam ${ }^{3}$ \\ ${ }^{1}$ Department of Pharmaceutical Sciences, Guru Jambheshwar University of Science and Technology, Hisar, Haryana 125001, India \\ ${ }^{2}$ Padmnabham Ayurveda Hospital and Research Center, 75 Jawahar Nagar Colony, Tonk Road, Jaipur, Rajasthan, India \\ ${ }^{3}$ M.S.M. Institute of Ayurveda, B.P.S. Mahila Vishwavidyalaya, Khanpur Kalan, Sonipat, Haryana, India \\ Correspondence should be addressed to Dinesh Dhingra; din_dhingra@rediffmail.com
}

Received 2 October 2013; Accepted 7 November 2013; Published 17 February 2014

Academic Editors: G. Edwards, R. Fantozzi, T. W. Stone, and S.-N. Wu

Copyright (C) 2014 Dinesh Dhingra et al. This is an open access article distributed under the Creative Commons Attribution License, which permits unrestricted use, distribution, and reproduction in any medium, provided the original work is properly cited.

\begin{abstract}
The present study was designed to investigate antihyperlipidemic activity of dried pulp of Aloe succotrina leaves in Wistar albino rats. Hyperlipidemia was induced in rats by feeding them high fat diet (HFD) or D-fructose (25\% w/v) for 4 successive weeks. From 15th to 28 th day, dried pulp (100 and $200 \mathrm{mg} / \mathrm{kg}$, p.o) and atorvastatin $(10 \mathrm{mg} / \mathrm{kg}$, p.o.) per se were administered $2 \mathrm{~h}$ prior to feeding rats with HFD or fructose. Aloe succotrina did not significantly decrease the body weight of rats. The dried pulp and atorvastatin per se significantly decreased relative liver weight but did not significantly affect relative heart weight. HFD or fructose significantly increased serum total cholesterol, triglycerides, LDL-c, and VLDL, and decreased HDL-c; significantly increased liver MDA and decreased GSH levels. The dried pulp ( $200 \mathrm{mg} / \mathrm{kg}$ p.o.) significantly reversed high fat diet-induced and fructose-induced hyperlipidemia and atherogenic index. Aloe succotrina significantly decreased HMG Co-A reductase activity. Antihyperlipidemic effect of the dried pulp was comparable to atorvastatin. Thus, Aloe succotrina produced significant antihyperlipidemic activity in both HFD and fructose-induced hyperlipidemic rats, possibly through normalization of serum lipid profile, HMG-CoA reductase inhibitory activity, and amelioration of oxidative stress in liver.
\end{abstract}

\section{Introduction}

Hyperlipidemia is a heterogeneous disorder commonly characterized by elevated serum total cholesterol, low density and very low-density lipoprotein cholesterol, triglycerides, and decreased high-density lipoprotein levels [1]. Hyperlipidemia is one of the greatest risk factors contributing to the prevalence and severity of atherosclerosis and subsequent coronary heart disease [2]. Liver synthesizes two-third of the total cholesterol made in the body. The rate limiting enzyme is 3-hydroxy-3-methylglutaryl (HMG)-Co A reductase and provides feedback regulation by controlling the cholesterol concentrations in cells. Treatment of hyperlipidemia involves diet control, exercise, and the use of lipid-lowering diets and drugs [3]. The most commonly employed drugs for treatment of hyperlipidemia include hydroxymethylglutarate coenzyme A (HMG-CoA) reductase inhibitors, also called as statins.
Other drugs employed for treatment of hyperlipidemia include bile acid sequestrants (anion-exchange resins) such as cholestyramine and colestipol; fibrates such as clofibrate, gemfibrozil, fenofibrate, ciprofibrate, and bezafibrate; niacin; cholesterol absorption inhibitors such as ezetimibe; and omega-3-fatty acids [4].

In spite of the availability of a number of drugs for treatment of hyperlipidemia, antihyperlipidemic therapy is still deprived of the efficiency, safety, and finally "cost." For example, there is a risk of sever muscle damage with statins, which are particularly well suited for lowering LDL [5]. Niacin, a good drug for lowering triglycerides, may cause hyperglycemia and may also cause liver damage [6]. Adverse reactions of Achilles tendon xanthomas have been reported after the addition of niacin and bile acid sequestrants to ongoing statin therapy in patients of hypercholesterolemia [7]. Adverse effects due to the use of fibrates often relate 
to the skeletal muscle, kidneys, or liver. Fenofibrate induced rhabdomyolysis which was complicated with acute renal failure [8]. Thus, there is still need for development of better antihyperlipidemic agents. Plant products are frequently considered to be less toxic and free from side effects than synthetic ones. There are a number of plants reported to possess antihyperlipidemic activity in clinical studies such as Allium sativum [9], Commiphora mukul [10], soy or Glycine max [11], Nigella sativa [12], and Plantago ovata [13].

Leaves of Aloe succotrina have been reported to possess antimalarial [14] and antidiabetic [15] activities. But effect of Aloe succotrina on hyperlipidemia has not been explored. So the aim of our study was to explore dried pulp of Aloe succotrina leaves on high fat diet- and fructose-induced hyperlipidemia in rats.

\section{Materials and Methods}

2.1. Experimental Animals. Wistar albino rats of either sex, weighing 60-100 g, were procured from Disease Free Small Animal House, Lala Lajpat Rai University of Veterinary and Animal Sciences, Hisar, Haryana. The animals were housed separately in groups of 6 per cage $(43 \times 27 \times 15 \mathrm{~cm})$ under laboratory conditions with alternating light and dark cycle of $12 \mathrm{~h}$ each. The animals had free access to food and water. The animals were acclimatized for at least five days before the start of experiments. The experimental protocol was approved by Institutional Animals Ethics Committee and animal care was taken as per the guidelines of Committee for the Purpose of Control and Supervision of Experiments on Animals (CPCSEA), Government. of India (Registration no. 0436).

2.2. Drugs and Chemicals. Atorvastatin (Ranbaxy Laboratories Ltd., Gurgaon), D-fructose, cholesterol pure, cholic acid, egg yolk powder, EDTA, n-butanol, acetic acid, tris buffer, hydrochloride, sodium lauryl sulphate, thiobarbituric acid, trichloroacetic acid, ethanol, carboxy methyl cellulose, potassium chloride, Ellman's reagent (5,5' -dithiobis-(2-nitrobenzoic acid), DTNB), pyridine, sodium hydroxide, sodium arsenate, perchloric acid, ferric chloride and hydroxyl amine hydrochloride (HiMedia Laboratories Pvt., Ltd., Mumbai) were used in the present study.

2.3. Plant Material. Dried pulp of Aloe succotrina leaves was procured from Padmnabham Ayurveda Hospital and Research Centre, Jawahar Nagar Colony, Tonk Road, Jaipur (Rajasthan, India).

2.4. Vehicle. Dried pulp of Aloe succotrina leaves and atorvastatin were separately suspended in $1 \% \mathrm{w} / \mathrm{v}$ CMC just before administration to the animals.

\subsection{Induction of Hyperlipidemia}

2.5.1. High Fat Diet-Induced Hyperlipidemia. High fat diet was administered for 28 successive days to induce hyperlipidemia in rats [16]. High fat diet consisted of normal feed
(78.8\%), cholesterol pure (1\%), cholic acid (0.2\%), lard (10\%) and egg yolk powder (10\%). Normal feed contained only freshly cooked dalia (coarsely grounded wheat). Normal and high fat diets were freshly prepared each day.

2.5.2. Fructose-Induced Hyperlipidemia. D-fructose (25\% $\mathrm{w} / \mathrm{v})$ in drinking water was administered for 28 successive days to induce hyperlipidemia in rats [24].

2.6. Experimental Protocol. Animals were divided into the following groups and each group comprised of 3-6 rats.

\subsubsection{Groups for High Fat Diet Induced-Hyperlipidemia Model}

Group $1(n=6)$. Rats were fed with normal diet for 14 successive days. From the 15 th day to the 28 th day, vehicle CMC ( $1 \% \mathrm{w} / \mathrm{v})$ was administered orally $2 \mathrm{~h}$ before feeding the animals with normal diet.

Group $2(n=6)$. High fat diet was administered for 14 consecutive days. From the 15 th day to the 28 th day, vehicle CMC ( $1 \% \mathrm{w} / \mathrm{v})$ was administered orally $2 \mathrm{~h}$ before feeding the animals with high fat diet.

Group $3(n=3)$. High fat diet was administered for 14 consecutive days. From the 15 th day to the 28 th day, atorvastatin $(10 \mathrm{mg} / \mathrm{kg}$, p.o.) was administered orally $2 \mathrm{~h}$ before feeding the animals with high fat diet.

Groups 4 and 5 ( $n=5$ each). High fat diet was administered for 14 consecutive days. From the 15th day to the 28th day, dried pulp of Aloe succotrina leaves $(100 \mathrm{mg} / \mathrm{kg}$ and $200 \mathrm{mg} / \mathrm{kg}$, p.o. resp.) was administered orally $2 \mathrm{~h}$ before feeding the animals with high fat diet.

\subsubsection{Groups for Fructose-Induced Hyperlipidemia Model}

Group $6(n=5)$. Rats were fed with normal diet for 14 successive days. From the 15 th day to the 28th day, vehicle CMC ( $1 \% \mathrm{w} / \mathrm{v})$ was administered orally $2 \mathrm{~h}$ before feeding the animals with normal diet.

Group $7(n=5)$. Fructose $(25 \% \mathrm{w} / \mathrm{v})$ was administered for 14 consecutive days. From the 15 th day to the 28 th day, vehicle CMC ( $1 \% \mathrm{w} / \mathrm{v})$ was administered orally $2 \mathrm{~h}$ before feeding the animals with fructose $(25 \% \mathrm{w} / \mathrm{v})$.

Group $8(n=5)$. Fructose $(25 \% \mathrm{w} / \mathrm{v})$ was administered for 14 consecutive days. From the 15 th day to the 28 th day, atorvastatin $(10 \mathrm{mg} / \mathrm{kg}$, p.o.) was administered orally $2 \mathrm{~h}$ before feeding the animals with fructose solution.

Groups 9 and 10 ( $n=5$ each). Fructose $(25 \%$ w/v) was administered for 14 consecutive days. From the 15 th day to the 28th day, dried pulp of Aloe succotrina leaves $(100 \mathrm{mg} / \mathrm{kg}$ and $200 \mathrm{mg} / \mathrm{kg}$, p.o., resp.) was administered orally $2 \mathrm{~h}$ before feeding the animals with fructose solution. 
2.7. Measurement of Body Weight, Relative Liver, and Heart Weight of Rats. Body weights of animals of all groups were measured every week. But relative liver and heart weight of the animals were measured on the 29th day after sacrificing the animals. Relative liver and heart weight were calculated by applying the following formula [17]:

$$
\text { Relative weight }=\left(\frac{\text { Organ weight }}{\text { Body weight }}\right) \times 100 \text {. }
$$

2.8. Biochemical Estimations in Serum. On day 29, animals were sacrificed by carotid artery bleeding under light anesthesia with diethyl ether and blood samples (about $0.5 \mathrm{~mL}$ from each animal) were collected. Blood was kept for $30 \mathrm{~min}$ for coagulation and then serum was separated by centrifugation at $3000 \mathrm{rpm}$ using centrifuge (Remi, Mumbai). Total cholesterol [18], HDL-cholesterol [19], and triglycerides [20] were measured in serum samples using commercially biochemical kits (Erba Diagnostics, Mannheim, Germany). The samples were analyzed by using semiautomatic autoanalyzer (Chem 5 plus $-\mathrm{V}_{2}$ autoanalyzer; Erba Mannheim, Germany).

\subsection{Biochemical Estimations in Liver Homogenate}

2.9.1. Estimation of Reduced Glutathione (GSH) Levels. Reduced glutathione levels were measured in liver homogenate by using the procedure as reported earlier [21]. The tissue sample (200 mg of liver) was homogenized in $8.0 \mathrm{~mL}$ of $0.02 \mathrm{M}$ EDTA in an ice bath. The homogenates were kept in the ice bath until used. Aliquots of $5.0 \mathrm{~mL}$ of the homogenates were mixed in $15.0 \mathrm{~mL}$ test tubes with $4.0 \mathrm{~mL}$ distilled water and $1.0 \mathrm{~mL}$ of $50 \%$ trichloroacetic acid (TCA). The tubes were centrifuged for $15 \mathrm{~min}$ at $3000 \mathrm{rpm}$. The supernatant $(2.0 \mathrm{~mL})$ was mixed with $4.0 \mathrm{~mL}$ of $0.4 \mathrm{M}$ Tris buffer ( $\mathrm{pH} 8.9$ ), followed by addition of $0.1 \mathrm{~mL}$ Ellman's reagent [5,5-dithiobis-(2-nitrobenzoicacid)] and the sample was shaken. The absorbance was read within $5 \mathrm{~min}$ of the addition of DTNB at $412 \mathrm{~nm}$ against a reagent blank with no homogenate, using double beam UVVisible spectrophotometer (Varian Cary 5000 UV-VIS-NIR Spectrophotometer, The Netherland).

2.9.2. Estimation of Malondialdehyde Levels. Malondialdehyde (MDA), an index of free radical generation/lipid peroxidation, was measured in liver homogenate by using the procedure as reported earlier [22]. The tissue sample (100 mg of liver) was homogenized in $9.0 \mathrm{~mL}$ of KCL. Briefly, the reaction mixture consisted of $0.2 \mathrm{~mL}$ of $8.1 \%$ sodium lauryl

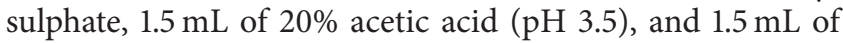
$0.8 \%$ aqueous solution of thiobarbituric acid added to $0.2 \mathrm{~mL}$ of liver homogenate. The mixture was made up to $4.0 \mathrm{~mL}$ with distilled water and heated at $95^{\circ} \mathrm{C}$ for $60 \mathrm{~min}$. After cooling the contents under running tap water, $5.0 \mathrm{~mL}$ of $\mathrm{n}$ butanol and pyridine $(15: 1 \mathrm{v} / \mathrm{v})$ and $1.0 \mathrm{~mL}$ of distilled water were added. The contents were centrifuged at about $4000 \mathrm{rpm}$ for $10 \mathrm{~min}$. The organic layer was separated out and its absorbance was measured at $532 \mathrm{~nm}$ using double beam UVVisible spectrophotometer (Varian Cary 5000 UV-VIS-NIR
Spectrophotometer, The Netherland) against a reagent blank. MDA values were calculated using the extinction coefficient of MDA-thiobarbituric acid complex $1.56 \times 10^{5} \mathrm{l} / \mathrm{mol} \times \mathrm{cm}$ and expressed as $\mathrm{nmol} / \mathrm{mg}$ tissue.

2.9.3. Estimation of HMG-CoA Reductase Activity (HMGCoA/Mevalonate Ratio). HMG-CoA reductase activity was measured in liver homogenate using the procedure of Venugopala Rao and Ramakrishnan [23]. The ratio of HMG-CoA to mevalonate was taken as an index of enzyme activity which catalyzes the conversion of HMG to mevalonate. The lower the ratio, the higher the enzyme activity. The liver sample $(100 \mathrm{mg})$ was homogenized in $1.0 \mathrm{~mL}$ of arsenate $(1 \mathrm{gm} / \mathrm{L})$ solution. Equal volumes $(0.5 \mathrm{~mL}$ each) of fresh liver $10 \%$ tissue homogenate and diluted perchloric acid $(50 \mathrm{~mL} / \mathrm{L})$ were mixed together. This was allowed to stand for 5 minute and centrifuged at about $2000 \mathrm{rpm}$ for $10 \mathrm{~min}$. This was filtered and $1 \mathrm{~mL}$ of filtrate was mixed with $0.5 \mathrm{~mL}$ of freshly prepared hydroxyl amine $(2 \mathrm{~mol} / \mathrm{L})$ reagent of $\mathrm{pH} 5.5$ for HMG-CoA and with $0.5 \mathrm{~mL}$ of freshly prepared hydroxyl amine $(2 \mathrm{~mol} / \mathrm{L})$ reagent of $\mathrm{pH} 2.1$ for mevalonate. After 5 minutes, $1.5 \mathrm{~mL}$ of ferric chloride reagent (prepared by dissolving $5.2 \mathrm{gm}$ of trichloroacetic acid and $10 \mathrm{gm}$ of ferric chloride in $50 \mathrm{~mL}$ of $0.65 \mathrm{~mol} / \mathrm{L} \mathrm{HCl}$ ) was added to each of the test tube for HMG-CoA and mevalonate. The tubes were shaken well. Absorbance was read after $10 \mathrm{~min}$ at $540 \mathrm{~nm}$ versus a similarly treated arsenate blank using double beam UV-Visible spectrophotometer (Varian Cary 5000 UV-VISNIR Spectrophotometer, The Netherland).

2.10. Statistical Analysis. All values were expressed as mean \pm SEM. The data were statistically analyzed using one-way ANOVA followed by Tukey-Kramer multiple comparison test. The $P<0.05$ was considered to be statistically significant.

\section{Results}

\subsection{Effects of Dried Pulp of Aloe succotrina Leaves on High Fat Diet-Induced Hyperlipidemic Rats}

3.1.1. Effect of Aloe succotrina on Body Weight. High fat diet significantly increased the body weight as compared to vehicle treated control after 2 weeks of treatment and this increase in body weight continued up to 4 weeks. Dried pulp of Aloe succotrina leaves (100 mg and $200 \mathrm{mg} / \mathrm{kg}$, p.o.) and atorvastatin per se administered from the 15 th day to the 28 th day did not significantly decrease the body weights of high fat diet-induced hyperlipidemic rats at the $3 \mathrm{rd}$ and 4 th weeks of treatment as compared to their respective high fat diet control (Table 1).

3.1.2. Effect of Aloe succotrina on Relative Liver and Heart Weight. High fat diet significantly increased the relative liver weight of rats but did not significantly increase the relative heart weight of animals as compared to vehicle treated control after 28 days. Dried pulp of Aloe succotrina leaves $(100 \mathrm{mg}$ and $200 \mathrm{mg} / \mathrm{kg}$ p.o.) and atorvastatin (10 mg/kg p.o.) per se 
TABLE 1: Effect of dried pulp of Aloe succotrina leaves on body weight of HFD-induced hyperlipidemic rats.

\begin{tabular}{|c|c|c|c|c|c|c|c|c|}
\hline \multirow{2}{*}{$\begin{array}{l}\text { Group } \\
\text { number }\end{array}$} & \multirow{2}{*}{$\begin{array}{l}\text { Treatment } \\
\text { (p.o.) }\end{array}$} & \multirow{2}{*}{$\begin{array}{l}\text { Dose } \\
\left(\mathrm{kg}^{-1}\right)\end{array}$} & \multirow{2}{*}{$\begin{array}{l}\text { Number } \\
\text { of animals }\end{array}$} & \multicolumn{5}{|c|}{ Body weight (g) } \\
\hline & & & & Week 0 & Week 1 & Week 2 & Week 3 & Week 4 \\
\hline 1 & $\begin{array}{c}\text { Vehicle } \\
(1 \% \text { w/v CMC) }\end{array}$ & $10 \mathrm{~mL}$ & 6 & $83.16 \pm 3.98$ & $87.66 \pm 4.09$ & $95.5 \pm 3.77$ & $103.33 \pm 4.82$ & $104.33 \pm 4.78$ \\
\hline 2 & $\begin{array}{c}\text { Vehicle } \\
(1 \% \text { w/v CMC) } \\
+ \text { HFD }\end{array}$ & $10 \mathrm{~mL}$ & 6 & $82.33 \pm 2.96$ & $102.16 \pm 3.19^{\mathrm{a}}$ & $112.66 \pm 4.73^{c}$ & $129.66 \pm 5.90^{c}$ & $136.00 \pm 5.75^{\mathrm{c}}$ \\
\hline 3 & $\begin{array}{l}\text { Atorvastatin }+ \\
\text { HFD }\end{array}$ & $10 \mathrm{mg}$ & 3 & $83.00 \pm 4.44$ & $98.16 \pm 5.16$ & $118.16 \pm 8.37$ & $124.00 \pm 8.09$ & $133.33 \pm 11.86$ \\
\hline 4 & $\begin{array}{c}\text { Aloe succotrina } \\
+ \text { HFD }\end{array}$ & $100 \mathrm{mg}$ & 5 & $79.16 \pm 3.92$ & $101.83 \pm 5.134$ & $124.5 \pm 5.74$ & $106.66 \pm 5.48$ & $115.8 \pm 16.33$ \\
\hline 5 & $\begin{array}{c}\text { Aloe succotrina } \\
+ \text { HFD }\end{array}$ & $200 \mathrm{mg}$ & 5 & $81.83 \pm 5.13$ & $104.00 \pm 7.17$ & $129.83 \pm 8.25$ & $138.83 \pm 6.98$ & $150.6 \pm 7.36$ \\
\hline
\end{tabular}

In each group; values are in Mean \pm SEM; data were analyzed by one-way ANOVA followed by Tukey-Kramer multiple comparison test.

$\mathrm{F}(4,25)=21.29 ; P<0.0001$ (for HFD treated control from week 0 to week 4).

$\mathrm{F}(4,25)=1.636 ; P<0.1964$ (comparison among various groups at week 1 ).

$\mathrm{F}(4,25)=4.207 ; P<0.0097$ (comparison among various groups at week 2 ).

$\mathrm{F}(4,25)=5.585 ; P<0.0024$ (comparison among various groups at week 3 ).

$\mathrm{F}(4,20)=7.901 ; P<0.0005$ (comparison among various groups at week 4 ).

${ }^{\mathrm{a}} P<0.05$ and ${ }^{\mathrm{c}} P<0.001$ as compared to HFD treated control (week 0 ).

TABLE 2: Effect of dried pulp of Aloe succotrina leaves on relative liver and heart weight of HFD-induced hyperlipidemic rats.

\begin{tabular}{lcccc}
\hline Group number & Treatment (p.o.) & Dose $\left(\mathrm{kg}^{-1}\right)$ & Number of animals & \multicolumn{2}{c}{$\begin{array}{c}\text { Relative organ weight } \\
\text { Liver weight }\end{array}$} \\
\hline 1 & Vehicle (1\% w/v CMC) & $10 \mathrm{~mL}$ & 6 & $4.98 \pm 0.32$ \\
2 & Vehicle (1\% w/v CMC) + HFD & $10 \mathrm{~mL}$ & 6 & $7.80 \pm 0.54^{\# \#}$ \\
3 & Atorvastatin + HFD & $10 \mathrm{mg}$ & 3 & $0.70 \pm 0.07$ \\
4 & Aloe succotrina + HFD & $100 \mathrm{mg}$ & 5 & 0.04 \\
5 & Aloe succotrina + HFD & $200 \mathrm{mg}$ & 5 & $5.87 \pm 0.31^{\mathrm{a}}$ \\
\hline
\end{tabular}

Relative weight $=($ organ weight/body weight $) \times 100$. Values are in Mean \pm SEM; data were analyzed by one-way ANOVA followed by Tukey-Kramer multiple comparison test.

$\mathrm{F}(4,20)=8.271 ; P<0.0004$ (for relative liver weight).

$\mathrm{F}(4,20)=0.747 ; P<0.5715$ (for relative heart weight).

${ }_{\# \# P} P<0.001$ as compared to vehicle treated control; ${ }^{\mathrm{a}} P<0.05$ as compared to HFD treated control.

administered from the 15th day to the 28th day significantly decreased relative liver weight as compared to high fat diet control on the 29th day (Table 2) but did not significantly affect relative heart weight (Table 2).

3.1.3. Effect of Aloe succotrina on Serum Lipid Parameters. High fat diet significantly increased serum triglycerides, total cholesterol, LDL-c, VLDL, and atherogenic index and significantly decreased HDL-cholesterol levels as compared to vehicle treated control. Dried pulp of Aloe succotrina leaves $(200 \mathrm{mg} / \mathrm{kg}$ p.o.) and atorvastatin $(10 \mathrm{mg} / \mathrm{kg})$ per se significantly decreased serum total cholesterol, triglycerides, VLDL, LDL-c, and atherogenic index and increased HDLcholesterol levels as compared to high fat diet control. Lower dose $(100 \mathrm{mg} / \mathrm{kg})$ of dried pulp of Aloe succotrina leavessignificantly decreased serum total cholesterol and LDL-c and increased HDL-cholesterol levels but did not significantly affect TG, VLDL, and atherogenic index as compared to high fat diet control (Table 3).
3.1.4. Effect of Aloe succotrina on Liver $H M G /$ Mevalonate Ratio, Malondialdehyde (MDA), and Reduced Glutathione (GSH) Levels. High fat diet significantly increased MDA levels and decreased GSH levels as compared to vehicle treated control. Dried pulp of Aloe succotrina leaves ( $200 \mathrm{mg} / \mathrm{kg}$ p.o.) and atorvastatin $(10 \mathrm{mg} / \mathrm{kg})$ per se significantly increased HMG/mevalonate ratio (or decreased HMG-CoA reductase activity), decreased MDA levels, and increased GSH levels as compared to high fat diet control. Lower dose $(100 \mathrm{mg} / \mathrm{kg})$ of dried pulp of Aloe succotrina leaves significantly decreased MDA levels and did not significantly affect GSH and HMGCoA reductase activity as compared to high fat diet control (Table 4).

\subsection{Effect of Dried Pulp of Aloe succotrina Leaves on Fructose- Induced Hyperlipidemic Rats}

3.2.1. Effect of Aloe succotrina on Body Weight. Fructose $(25 \% \mathrm{w} / \mathrm{v})$, dried pulp of Aloe succotrina leaves $(100 \mathrm{mg}$ and $200 \mathrm{mg} / \mathrm{kg}$ p.o.), and atorvastatin (10 mg/kg p.o.) per se did 
TABLE 3: Effect of dried pulp of Aloe succotrina leaves on serum lipid levels in HFD-induced hyperlipidemic rats.

\begin{tabular}{|c|c|c|c|c|c|c|c|c|}
\hline \multirow[b]{2}{*}{$\begin{array}{l}\text { Group } \\
\text { number }\end{array}$} & \multirow[b]{2}{*}{$\begin{array}{l}\text { Treatment } \\
\text { (p.o.) }\end{array}$} & \multirow[b]{2}{*}{$\begin{array}{l}\text { Dose } \\
\left(\mathrm{kg}^{-1}\right)\end{array}$} & \multicolumn{6}{|c|}{ Lipid levels (mg/dL) } \\
\hline & & & TC & TG & HDL-c & VLDL & LDL-c & $\begin{array}{c}\mathrm{AI} \\
(\mathrm{TG} / \mathrm{HDL})\end{array}$ \\
\hline 1 & $\begin{array}{c}\text { Vehicle } \\
(1 \% \text { w/v CMC }) \\
(n=6)\end{array}$ & $10 \mathrm{~mL}$ & $78.24 \pm 4.08$ & $19.55 \pm 5.04$ & $37.18 \pm 0.45$ & $3.91 \pm 1.01$ & $36.99 \pm 4.64$ & $0.28 \pm 0.07$ \\
\hline 2 & $\begin{array}{c}\text { Vehicle } \\
(1 \% \text { w/v CMC) } \\
+\operatorname{HFD~}(n=6)\end{array}$ & $10 \mathrm{~mL}$ & $315.42 \pm 27.17^{\# \# \#}$ & $108.04 \pm 11.67^{\# \# \#}$ & $17.38 \pm 3.97^{\# \# \#}$ & $21.60 \pm 2.34^{\# \# \#}$ & $276.40 \pm 26.38^{\# \# \#}$ & $10.57 \pm 3.86^{\#}$ \\
\hline 3 & $\begin{array}{l}\text { Atorvastatin }+ \\
\operatorname{HFD}(n=3)\end{array}$ & $10 \mathrm{mg}$ & $156.06 \pm 20.65^{c}$ & $28.42 \pm 6.65^{\mathrm{b}}$ & $50.37 \pm 3.67^{\mathrm{c}}$ & $5.68 \pm 1.33^{\mathrm{b}}$ & $100.00 \pm 20.36^{\mathrm{c}}$ & $0.55 \pm 0.09^{\mathrm{a}}$ \\
\hline 4 & $\begin{array}{l}\text { Aloe succotrina } \\
+\operatorname{HFD}(n=5)\end{array}$ & $100 \mathrm{mg}$ & $231.32 \pm 22.00^{\mathrm{a}}$ & $94.90 \pm 21.38$ & $33.94 \pm 3.30^{\mathrm{b}}$ & $18.98 \pm 4.28$ & $178.45 \pm 22.40^{\mathrm{b}}$ & $2.84 \pm 0.63$ \\
\hline 5 & $\begin{array}{l}\text { Aloe succotrina } \\
+\operatorname{HFD}(n=5)\end{array}$ & $200 \mathrm{mg}$ & $181.30 \pm 12.26^{\mathrm{c}}$ & $48.34 \pm 9.66^{\mathrm{a}}$ & $47.65 \pm 1.24^{\mathrm{c}}$ & $9.67 \pm 1.93^{\mathrm{a}}$ & $123.98 \pm 11.70^{c}$ & $1.02 \pm 0.22^{\mathrm{a}}$ \\
\hline
\end{tabular}

Values are in Mean \pm SEM; data was analyzed by one-way ANOVA followed by Tukey-Kramer multiple comparison test. TC-total cholesterol; TGtriglycerides; HDL-c-high density lipoprotein-cholesterol; LDL-c-low density lipoprotein-cholesterol; VLDL-very low density lipoprotein- cholesterol; $\mathrm{AI}-$ atherogenic index.

$\mathrm{F}(4,20)=25.856 ; P<0.0001$ (for total cholesterol).

$\mathrm{F}(4,20)=10.308 ; P<0.0001$ (for triglycerides).

$\mathrm{F}(4,20)=21.007 ; P<0.0001$ (for HDL-c).

$\mathrm{F}(4,20)=10.297 ; P<0.0001($ for VLDL).

$\mathrm{F}(4,20)=27.085 ; P<0.0001$ (for LDL-c).

$\mathrm{F}(4,20)=4.623 ; P<0.0083$ (for $\mathrm{AI}$ ).

${ }^{\#} P<0.05$ and ${ }^{\# \# \#} P<0.001$ as compared to vehicle treated control; ${ }^{\mathrm{a}} P<0.05,{ }^{\mathrm{b}} P<0.01$, and ${ }^{\mathrm{c}} P<0.001$ as compared to HFD treated control.

TABLE 4: Effect of dried pulp of Aloe succotrina leaves on liver HMG/mevalonate ratio, malondialdehyde (MDA), and reduced glutathione (GSH) levels of HFD-induced hyperlipidemic rats.

\begin{tabular}{|c|c|c|c|c|c|c|}
\hline $\begin{array}{l}\text { Group } \\
\text { number }\end{array}$ & Treatment (p.o.) & Dose $\left(\mathrm{kg}^{-1}\right)$ & Number of animals & $\begin{array}{l}\text { HMG/mevalonate } \\
\text { ratio }\end{array}$ & $\begin{array}{c}\text { MDA levels } \\
\text { (nmol/mg tissue) }\end{array}$ & $\begin{array}{c}\text { GSH levels } \\
(\mu \mathrm{mol} / \mathrm{mg} \text { tissue })\end{array}$ \\
\hline 1 & $\begin{array}{c}\text { Vehicle } \\
\text { (1\% w/v CMC) }\end{array}$ & $10 \mathrm{~mL}$ & 6 & $0.59 \pm 0.23$ & $52.60 \pm 10.49$ & $735.21 \pm 57.31$ \\
\hline 2 & $\begin{array}{c}\text { Vehicle } \\
(1 \% \text { w/v CMC })+ \\
\text { HFD }\end{array}$ & $10 \mathrm{~mL}$ & 6 & $0.37 \pm 0.13$ & $179.74 \pm 23.77^{\# \#}$ & $297.62 \pm 24.81^{\# \# \#}$ \\
\hline 3 & Atorvastatin + HFD & $10 \mathrm{mg}$ & 3 & $3.29 \pm 0.37^{\mathrm{c}}$ & $13.43 \pm 4.65^{\mathrm{c}}$ & $665.84 \pm 24.34^{\mathrm{c}}$ \\
\hline 4 & $\begin{array}{c}\text { Aloe succotrina }+ \\
\text { HFD }\end{array}$ & $100 \mathrm{mg}$ & 5 & $0.81 \pm 0.13$ & $78.56 \pm 27.66^{b}$ & $303.84 \pm 31.85$ \\
\hline 5 & $\begin{array}{c}\text { Aloe succotrina }+ \\
\text { HFD }\end{array}$ & $200 \mathrm{mg}$ & 5 & $1.68 \pm 0.34^{\mathrm{b}}$ & $52.72 \pm 12.32^{c}$ & $617.86 \pm 26.35^{\mathrm{c}}$ \\
\hline
\end{tabular}

Values are in Mean \pm SEM; data was analyzed by one-way ANOVA followed by Tukey-Kramer multiple comparison test.

$\mathrm{F}(4,20)=14.402 ; P<0.0001$ (for HMG/mevalonate ratio).

$\mathrm{F}(4,20)=10.117 ; P<0.0001$ (for MDA levels).

$\mathrm{F}(4,20)=25.390 ; P<0.0001$ (for reduced glutathione levels).

${ }^{\# \#} P<0.01$ and ${ }^{\# \#} P<0.001$ as compared to vehicle treated control; ${ }^{\mathrm{b}} P<0.05$ and ${ }^{\mathrm{c}} P<0.001$ as compared to HFD treated control.

not significantly affect the body weight of rats as compared to vehicle treated control (Table 5).

3.2.2. Effect of Aloe succotrina on Relative Liver and Heart Weight. Fructose $(25 \% \mathrm{w} / \mathrm{v})$ significantly increased the relative liver weight of animals but did not significantly increase the relative heart weight of animals as compared to vehicle treated control after 28 days. Dried pulp of Aloe succotrina leaves (100 $\mathrm{mg}$ and $200 \mathrm{mg} / \mathrm{kg}$ p.o.) and atorvastatin $(10 \mathrm{mg} / \mathrm{kg}$ p.o.) per se significantly decreased relative liver weight of animals as compared to fructose treated control but did not significantly affect relative heart weight of animals (Table 6).

3.2.3. Effect of Aloe succotrina on Serum Lipid Parameters. Fructose $(25 \% \mathrm{w} / \mathrm{v})$ significantly increased serum total cholesterol, triglycerides, VLDL, LDL-c, and atherogenic index and significantly decreased HDL-cholesterol levels as compared to vehicle treated control. Dried pulp of Aloe succotrina leaves $(200 \mathrm{mg} / \mathrm{kg}$ p.o. $)$ and atorvastatin $(10 \mathrm{mg} / \mathrm{kg}$ p.o.) per se significantly decreased serum total cholesterol, triglycerides, VLDL, LDL-c, and atherogenic index and 
TABLE 5: Effect of dried pulp of Aloe succotrina leaves on body weight of fructose-induced hyperlipidemic rats.

\begin{tabular}{|c|c|c|c|c|c|c|c|}
\hline \multirow{2}{*}{ Group number } & \multirow{2}{*}{ Treatment (p.o.) } & \multirow{2}{*}{ Dose $\left(\mathrm{kg}^{-1}\right)$} & \multicolumn{5}{|c|}{ Body weight (g) } \\
\hline & & & Week 0 & Week 1 & Week 2 & Week 3 & Week 4 \\
\hline 1 & $\begin{array}{c}\text { Vehicle } \\
(1 \% \mathrm{w} / \mathrm{v} \text { CMC }) 0\end{array}$ & $10 \mathrm{~mL}$ & $80.66 \pm 4.39$ & $83.83 \pm 5.43$ & $94.00 \pm 4.97$ & $102.33 \pm 4.69$ & $114.16 \pm 5.52$ \\
\hline 2 & $\begin{array}{c}\text { Vehicle } \\
(1 \% \mathrm{w} / \mathrm{v} \text { CMC })+ \\
\text { fructose }(25 \% \mathrm{w} / \mathrm{v})\end{array}$ & $10 \mathrm{~mL}$ & $81.16 \pm 7.35$ & $88.16 \pm 9.00$ & $92.00 \pm 10.34$ & $95.16 \pm 10.42$ & $106.83 \pm 11.08$ \\
\hline 3 & $\begin{array}{c}\text { Atorvastatin }+ \\
\text { fructose }(25 \% \mathrm{w} / \mathrm{v})\end{array}$ & $10 \mathrm{mg}$ & $78.66 \pm 3.54$ & $86.83 \pm 4.33$ & $95.66 \pm 4.75$ & $102.83 \pm 3.52$ & $113.16 \pm 3.09$ \\
\hline 4 & $\begin{array}{l}\text { Aloe succotrina }+ \\
\text { fructose }(25 \% \mathrm{w} / \mathrm{v})\end{array}$ & $100 \mathrm{mg}$ & $83.16 \pm 5.40$ & $91.66 \pm 7.19$ & $99.83 \pm 7.04$ & $96.83 \pm 6.56$ & $106.8 \pm 9.50$ \\
\hline 5 & $\begin{array}{c}\text { Aloe succotrina }+ \\
\text { fructose }(25 \% \mathrm{w} / \mathrm{v})\end{array}$ & $200 \mathrm{mg}$ & $80.83 \pm 4.47$ & $86.5 \pm 6.13$ & $96.16 \pm 5.50$ & $106.00 \pm 4.44$ & $119.16 \pm 6.14$ \\
\hline
\end{tabular}

$(n=5)$ in each group. Values are in Mean \pm SEM; data was analyzed by one-way ANOVA followed by Tukey-Kramer multiple comparison test.

$\mathrm{F}(4,25)=0.95 ; P<0.04516$ (for fructose treated control from week 0 to week 4 ).

$\mathrm{F}(4,25)=0.19 ; P<0.9431$ (comparison among various groups at week 1$)$.

$\mathrm{F}(4,25)=0.18 ; P<0.9464$ (comparison among various groups at week 2 ).

$\mathrm{F}(4,25)=0.49 ; P<0.7422$ (comparison among various groups at week 3 ).

$\mathrm{F}(4,25)=0.49 ; P<0.7432$ (comparison among various groups at week 4$)$.

TABLE 6: Effect of dried pulp of Aloe succotrina leaves on relative liver and heart weight of fructose-induced hyperlipidemic rats.

\begin{tabular}{|c|c|c|c|c|}
\hline \multirow{2}{*}{ Group number } & \multirow{2}{*}{ Treatment (p.o.) } & \multirow{2}{*}{ Dose $\left(\mathrm{kg}^{-1}\right)$} & \multicolumn{2}{|c|}{ Relative organ weight } \\
\hline & & & Liver weight & Heart weight \\
\hline 1 & Vehicle (1\% w/v CMC) & $10 \mathrm{~mL}$ & $3.48 \pm 0.15$ & $0.40 \pm 0.01$ \\
\hline 2 & Vehicle $(1 \% \mathrm{w} / \mathrm{v} \mathrm{CMC})+$ fructose $(25 \% \mathrm{w} / \mathrm{v})$ & $10 \mathrm{~mL}$ & $5.78 \pm 0.74^{\# \#}$ & $0.42 \pm 0.02$ \\
\hline 3 & Atorvastatin + fructose $(25 \% \mathrm{w} / \mathrm{v})$ & $10 \mathrm{mg}$ & $3.87 \pm 0.36^{\mathrm{a}}$ & $0.47 \pm 0.03$ \\
\hline 4 & Aloe succotrina + fructose $(25 \% \mathrm{w} / \mathrm{v})$ & $100 \mathrm{mg}$ & $3.92 \pm 0.30^{\mathrm{a}}$ & $0.46 \pm 0.02$ \\
\hline 5 & Aloe succotrina + fructose $(25 \% \mathrm{w} / \mathrm{v})$ & $200 \mathrm{mg}$ & $3.87 \pm 0.29^{\mathrm{a}}$ & $0.49 \pm 0.03$ \\
\hline
\end{tabular}

Relative weight $=($ organ weight $/$ body weight $) \times 100$.

$(n=5)$ in each group. Values are in Mean \pm SEM; data was analyzed by one-way ANOVA followed by Tukey-Kramer multiple comparison test.

$\mathrm{F}(4,21)=4.742 ; P<0.007$ (for relative liver weight).

$\mathrm{F}(4,21)=1.594 ; P<0.1045$ (for relative heart weight).

${ }^{\# \#} P<0.01$ as compared to vehicle treated control; ${ }^{a} P<0.05$ as compared to fructose treated control.

increased HDL-cholesterol levels as compared to fructose treated control on the 29th day. Lower dose $(100 \mathrm{mg} / \mathrm{kg})$ of the dried pulp significantly decreased serum triglycerides, VLDL, and atherogenic index but did not significantly affect total cholesterol, LDL-c, and HDL-c as compared to fructose treated control (Table 7).

3.2.4. Effect of Aloe succotrina on Liver $H M G /$ Mevalonate Ratio, Malondialdehyde (MDA), and Reduced Glutathione (GSH) Levels. Fructose (25\% w/v) diet significantly increased MDA, and decreased GSH levels as compared to vehicle treated control. Dried pulp of Aloe succotrina leaves $(200 \mathrm{mg} / \mathrm{kg}$ p.o.) and atorvastatin $(10 \mathrm{mg} / \mathrm{kg})$ per se significantly increased $\mathrm{HMG} /$ mevalonate ratio and GSH levels and decreased MDA levels as compared to fructose treated control. Lower dose $(100 \mathrm{mg} / \mathrm{kg})$ of the dried pulp significantly decreased MDA levels but did not significantly affect GSH and HMG-CoA reductase activity as compared to fructose treated control (Table 8).

\section{Discussion}

In the present study, dried pulp of Aloe succotrina leaves produced significant antihyperlipidemic effect in high fat diet- and fructose-induced hyperlipidemic rats. Rats fed with HFD or fructose for 4 successive weeks induced significant hyperlipidemia, as indicated by the significant increase in serum levels of TC, TG, LDL-c, and VLDL and decrease in serum levels of HDL-c. This is supported by earlier studies $[17,24,25]$. High fat diet had unfavorable effect on body weight and relative liver weight but did not significantly affect relative heart weight. This is supported by earlier study where HFD increased body weight and relative liver weight without significantly affecting relative heart weight [26]. D-fructose $(25 \% \mathrm{w} / \mathrm{v})$ produced increase in relative liver weight, without significantly affecting body weight and relative heart weight of animals. This result is also supported by the literature [17].

Fructose has been reported to induce hypertriglyceridemia associated with insulin resistance, hyperinsulinemia, and hypertension. After the absorption in GIT, fructose 
TABLE 7: Effect of the dried pulp of Aloe succotrina leaves on serum lipid levels in fructose-induced hyperlipidemic rats.

\begin{tabular}{|c|c|c|c|c|c|c|c|c|}
\hline \multirow{2}{*}{$\begin{array}{l}\text { Group } \\
\text { number }\end{array}$} & \multirow{2}{*}{$\begin{array}{l}\text { Treatment } \\
\text { (p.o.) }\end{array}$} & \multirow{2}{*}{$\begin{array}{l}\text { Dose } \\
\left(\mathrm{kg}^{-1}\right)\end{array}$} & \multicolumn{6}{|c|}{ Lipid Levels (mg/dL) } \\
\hline & & & TC & TG & HDL-c & VLDL & LDL-c & $\begin{array}{c}\text { AI } \\
(\mathrm{TG} / \mathrm{HDL})\end{array}$ \\
\hline 1 & $\begin{array}{c}\text { Vehicle } \\
(1 \% \text { w/v CMC) }\end{array}$ & $10 \mathrm{~mL}$ & $67.09 \pm 2.74$ & $39.47 \pm 1.75$ & $37.26 \pm 1.83$ & $7.93 \pm 0.37$ & $21.83 \pm 4.54$ & $1.07 \pm 0.06$ \\
\hline 2 & $\begin{array}{c}\text { Vehicle } \\
\text { (1\% w/v CMC) } \\
+ \text { fructose } \\
(25 \% \mathrm{w} / \mathrm{v})\end{array}$ & $10 \mathrm{~mL}$ & $85.56 \pm 4.37^{\#}$ & $67.10 \pm 12.69^{\#}$ & $17.36 \pm 1.49^{\#}$ & $13.42 \pm 2.54^{\#}$ & $54.80 \pm 6.03^{\# \#}$ & $3.93 \pm 0.74^{\# \# \#}$ \\
\hline 3 & $\begin{array}{c}\text { Atorvastatin + } \\
\text { fructose } \\
(25 \% \mathrm{w} / \mathrm{v})\end{array}$ & $10 \mathrm{mg}$ & $64.32 \pm 4.09^{\mathrm{a}}$ & $22.59 \pm 2.14^{\mathrm{c}}$ & $41.72 \pm 6.06^{\mathrm{b}}$ & $4.52 \pm 0.43^{\mathrm{c}}$ & $18.09 \pm 4.89^{\mathrm{b}}$ & $0.60 \pm 0.10^{c}$ \\
\hline 4 & $\begin{array}{l}\text { Aloe succotrina } \\
\text { + fructose } \\
(25 \% \mathrm{w} / \mathrm{v})\end{array}$ & $100 \mathrm{mg}$ & $71.97 \pm 6.12$ & $33.22 \pm 3.98^{\mathrm{b}}$ & $42.33 \pm 5.48$ & $6.64 \pm 0.80^{\mathrm{b}}$ & $38.53 \pm 6.94$ & $1.42 \pm 0.32^{\mathrm{c}}$ \\
\hline 5 & $\begin{array}{c}\text { Aloe succotrina } \\
\text { + fructose } \\
(25 \% \mathrm{w} / \mathrm{v})\end{array}$ & $200 \mathrm{mg}$ & $66.15 \pm 2.16^{\mathrm{a}}$ & $24.74 \pm 2.44^{\mathrm{c}}$ & $26.80 \pm 4.71^{b}$ & $4.95 \pm 0.49^{c}$ & $18.87 \pm 5.27^{\mathrm{b}}$ & $0.62 \pm 0.09^{c}$ \\
\hline
\end{tabular}

$(n=5)$ in each group; values are in Mean \pm SEM; data was analyzed by one-way ANOVA followed by Tukey-Kramer multiple comparison test.

TC-total cholesterol; TG-triglycerides; HDL-c-high density lipoprotein- cholesterol.

LDL-c-low density lipoprotein- cholesterol; VLDL-very low density lipoprotein- cholesterol; AI-atherogenic index.

$\mathrm{F}(4,21)=4.344 ; P<0.0109$ (for total cholesterol); F $(4,21)=8.425 ; P<0.0004$ (for triglycerides); F $(4,21)=6.170 ; P<0.0021$ (for HDL-c); F (4, 21) = 8.414; $P<0.0004$ (for VLDL); F $(4,21)=8.118 ; P<0.0005$ (for LDL-c); F $(4,21)=14.361 ; P<0.0001$ (for AI).

${ }^{\#} P<0.05,{ }^{\# \#} P<0.01$, and ${ }^{\# \#} P<0.001$ as compared to vehicle treated control; ${ }^{\mathrm{a}} P<0.05,{ }^{\mathrm{b}} P<0.01$, and ${ }^{\mathrm{c}} P<0.001$ as compared to fructose treated control.

TABLE 8: Effect of dried pulp of Aloe succotrina leaves on liver HMG/mevalonate ratio, MDA, and reduced glutathione (GSH) levels of fructoseinduced hyperlipidemic rats.

\begin{tabular}{|c|c|c|c|c|c|}
\hline $\begin{array}{l}\text { Group } \\
\text { number }\end{array}$ & $\begin{array}{c}\text { Treatment } \\
\text { (p.o.) }\end{array}$ & $\begin{array}{l}\text { Dose } \\
\left(\mathrm{kg}^{-1}\right)\end{array}$ & $\begin{array}{l}\text { HMG } / \text { mevalonate } \\
\text { ratio }\end{array}$ & $\begin{array}{c}\text { MDA levels (nmol/mg } \\
\text { tissue) }\end{array}$ & $\begin{array}{c}\text { GSH levels }(\mu \mathrm{mol} / \mathrm{mg} \\
\text { tissue })\end{array}$ \\
\hline 1 & $\begin{array}{c}\text { Vehicle } \\
(1 \% \mathrm{w} / \mathrm{v} \mathrm{CMC})\end{array}$ & $10 \mathrm{~mL}$ & $3.44 \pm 1.22$ & $7.64 \pm 1.56$ & $524.55 \pm 35.89$ \\
\hline 2 & $\begin{array}{c}\text { Vehicle } \\
(1 \% \mathrm{w} / \mathrm{v} \text { CMC })+ \\
\text { fructose }(25 \% \mathrm{w} / \mathrm{v})\end{array}$ & $10 \mathrm{~mL}$ & $1.98 \pm 0.36$ & $49.02 \pm 0.92^{\# \# \#}$ & $317.20 \pm 19.40^{\# \# \#}$ \\
\hline 3 & $\begin{array}{c}\text { Atorvastatin }+ \\
\text { fructose }(25 \% \mathrm{w} / \mathrm{v})\end{array}$ & $10 \mathrm{mg}$ & $6.17 \pm 0.93^{\mathrm{a}}$ & $6.92 \pm 0.69^{c}$ & $445.74 \pm 19.57^{\mathrm{a}}$ \\
\hline 4 & $\begin{array}{c}\text { Aloe succotrina }+ \\
\text { fructose }(25 \% \mathrm{w} / \mathrm{v})\end{array}$ & $100 \mathrm{mg}$ & $3.75 \pm 0.78$ & $31.66 \pm 8.33^{\mathrm{a}}$ & $324.04 \pm 13.31$ \\
\hline 5 & $\begin{array}{l}\text { Aloe succotrina }+ \\
\text { fructose }(25 \% \mathrm{w} / \mathrm{v})\end{array}$ & $200 \mathrm{mg}$ & $5.66 \pm 0.67^{\mathrm{a}}$ & $8.85 \pm 2.05^{\mathrm{c}}$ & $428.13 \pm 34.29^{\mathrm{a}}$ \\
\hline
\end{tabular}

$(n=5)$ in each group; values are in Mean \pm SEM; data was analyzed by one-way ANOVA followed by Tukey-Kramer multiple comparison test.

$\mathrm{F}(4,21)=4.140 ; P<0.0001$ (for HMG/mevalonate ratio).

$\mathrm{F}(4,21)=7.607 ; P<0.0007$ (for MDA levels).

$\mathrm{F}(4,21)=11.289 ; P<0.0001$ (for reduced glutathione).

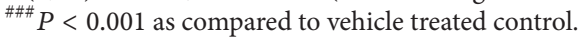

${ }^{\mathrm{a}} P<0.05,{ }^{\mathrm{b}} \mathrm{P}<0.05$, and ${ }^{\mathrm{c}} \mathrm{P}<0.001$ as compared to fructose treated control.

is transported via portal circulation to the liver, where it enters hepatocytes via the glucose transporter GLUT-5 independently of insulin and is rapidly metabolised. Fructose is metabolised into "glycerol-3-phosphate" and "acetyl CoA." These two intermediate metabolites are then used as substrates for glycerides synthesis, contributing to VLDLTG production in liver. The exposure of liver to such large quantities of fructose leads to rapid stimulation of lipogenesis and triglyceride accumulation which in turn contributes to reduced insulin sensitivity and hepatic insulin resistance/glucose intolerance $[25,27]$.
In both HFD and fructose-induced hyperlipidemia models, dried pulp of Aloe succotrina leaves ( $200 \mathrm{mg} / \mathrm{kg}$ p.o.) significantly decreased total cholesterol, triglycerides, LDL$c$, VLDL, atherogenic index, and liver weight and increased HDL-c levels. Antihyperlipidemic effect of the dried pulp was comparable to atorvastatin, a standard antihyperlipidemic drug (a HMG-CoA reductase inhibitor).

Hyperlipidemia is thought to increase cholesterol content, and this in turn leads to generation of reactive oxygen species (ROS), increase in lipid peroxidation [28] and decrease in activity of reduced glutathione. It has been 
reported that overproduction of ROS can induce cellular damage via oxidation of critical cellular components such as membrane lipids, proteins, and DNA [29]. The increased levels of total cholesterol in blood could induce arterial endothelial dysfunction, and vascular endothelial injury is the initial factor in atherosclerosis. Therefore, lowering lipid and protecting vascular endothelium play significant roles in preventing atherosclerosis [30].

Previous studies showed that high fat diet [31] or fructose [25] enhanced hepatic oxidative damage due to hepatic stress, resulting from the burden of high fat diet or fructose metabolism. In the present study, HFD or fructose significantly increased liver MDA levels and decreased GSH levels, indicating enhancement of oxidative stress. These findings are in agreement with earlier reports [32]. Dried pulp of Aloe succotrina leaves $(200 \mathrm{mg} / \mathrm{kg})$ significantly increased liver GSH levels and decreased MDA levels in both HFD and fructose-induced hyperlipidemic rats, thus reduced oxidative stress.

To identify the possible mechanism of the antihyperlipidemic effect of the dried pulp more specifically, the activity of HMG-CoA reductase was determined. Cholesterol homeostasis is maintained by the two processes, namely, cholesterol biosynthesis in which HMG-CoA reductase catalyzes rate limiting process and cholesterol absorption of both dietary cholesterol and cholesterol cleared from the liver through biliary secretion [33]. The HMG-CoA/mevalonate ratio has an inverse relationship to the activity HMG-CoA reductase. The result of the present study indicated that the activity of HMG-CoA reductase is significantly inhibited by the dried pulp of Aloe succotrina leaves $(200 \mathrm{mg} / \mathrm{kg})$ in both HFD and fructose-induced hyperlipidemic rats.

Hyperlipidemia is a well-known risk factor for atherosclerosis, which is a major contributor to the pathogenesis of heart and vascular diseases. Elevated blood concentration of cholesterol, especially in LDL-c, constitutes the key risk factor for atherosclerosis [34]. Atherogenic index indicates the deposition of foam cells or plaque or fatty infiltration or lipids in heart, coronaries, aorta, liver, and kidneys. The higher the atherogenic index, the higher the risk of the above organs for oxidative damage [35]. Aloe succotrina leaves $(200 \mathrm{mg} / \mathrm{kg})$ also markedly lowered atherogenic index in both HFD and fructose-induced hyperlipidemic rats. Thus, it indicated its potential in preventing atherosclerosis.

\section{Conclusions}

Dried pulp of Aloe succotrina leaves produced significant antihyperlipidemic activity in both HFD and fructoseinduced hyperlipidemic rats, possibly through normalization of serum lipid profile, HMG-CoA reductase inhibitory activity, and amelioration of oxidative stress in liver. Moreover, it also significantly decreased atherogenic index; thus, it might possess potential in preventing atherosclerosis. Further studies are required to isolate the active constituents involved in antihyperlipidemic activity of Aloe succotrina. Thus, dried pulp of Aloe succotrina leaves may be explored for its potential in the prevention and treatment of clinical hyperlipidemia.

\section{Conflict of Interests}

The authors do not have any conflict of interests regarding the content of this research paper.

\section{Acknowledgment}

The authors are thankful to Ranbaxy Laboratories Pvt., Ltd., Gurgaon (Haryana, India), for providing gift sample of atorvastatin.

\section{References}

[1] G. D. Kolovou, K. K. Anagnostopoulou, and D. V. Cokkinos, "Pathophysiology of dyslipidaemia in the metabolic syndrome," Postgraduate Medical Journal, vol. 81, no. 956, pp. 358-366, 2005.

[2] R. M. Krauss and Y. A. Kesaniemi, "Cardiovascular disease and hyperlipidaemia," Current Opinion in Lipidology, vol. 5, no. 4, pp. 249-251, 1994.

[3] N. J. Stone, "Lipid management: current diet and drug treatment options," The American Journal of Medicine, vol. 101, no. 4, supplement 1, pp. 40S-49S, 1996.

[4] Y. Lin, S. S. Mousa, N. Elshourbagy, and S. A. Mousa, "Current status and future directions in lipid management: emphasizing low-density lipoproteins, high-density lipoproteins, and triglycerides as targets for therapy," Vascular Health and Risk Management, vol. 6, no. 1, pp. 73-85, 2010.

[5] M. Kobayashi, T. Kagawa, K. Narumi, S. Itagaki, T. Hirano, and K. Iseki, "Bicarbonate supplementation as a preventive way in statins-induced muscle damage," Journal of Pharmacy and Pharmaceutical Sciences, vol. 11, no. 1, pp. 1-8, 2008.

[6] J. R. Guyton and H. E. Bays, "Safety considerations with niacin therapy," The American Journal of Cardiology, vol. 99, no. 6, pp. S22-S31, 2007.

[7] W. C. Lakey, N. Greyshock, and J. R. Guyton, "Adverse reactions of Achilles tendon xanthomas in three hypercholesterolemic patients after treatment intensification with niacin and bile acid sequestrants," Journal of Clinical Lipidology, vol. 7, no. 2, pp. 178181, 2013.

[8] M. Kiskac, M. Zorlu, M. Cakirca et al., "A case of rhabdomyolysis complicated with acute renal failure after resumption of fenofibrate therapy: a first report," Indian Journal of Pharmacology, vol. 45, no. 3, pp. 305-306, 2013.

[9] Y.-Y. Yeh and L. Liu, "Cholesterol-lowering effect of garlic extracts and organosulfur compounds: human and animal studies," Journal of Nutrition, vol. 131, no. 3, pp. 989S-993S, 2001.

[10] R. B. Singh, M. A. Niaz, and S. Ghosh, "Hypolipidemic and antioxidant effects of commiphora mukul as an adjunct to dietary therapy in patients with hypercholesterolemia," Cardiovascular Drugs and Therapy, vol. 8, no. 4, pp. 659-664, 1994.

[11] W. W. Wong, E. O’Brian Smith, J. E. Stuff, D. L. Hachey, W. C. Heird, and H. J. Pownell, "Cholesterol-lowering effect of soy protein in normocholesterolemic and hypercholesterolemic men," The American Journal of Clinical Nutrition, vol. 68, no. 6, pp. 13855S-13895S, 1998.

[12] A. M. Sabzghabaee, M. Dianatkhah, N. Sarrafzadegan, S. Asgary, and A. Ghannadi, "Clinical evaluation of Nigella sativa seeds for the treatment of hyperlipidemia: a randomized, placebo controlled clinical trial," Medical Archives, vol. 66, no. 3, pp. 198-200, 2012. 
[13] R. Solà, E. Bruckert, R.-M. Valls et al., "Soluble fibre (Plantago ovata husk) reduces plasma low-density lipoprotein (LDL) cholesterol, triglycerides, insulin, oxidised LDL and systolic blood pressure in hypercholesterolaemic patients: a randomised trial," Atherosclerosis, vol. 211, no. 2, pp. 630-637, 2010.

[14] R. L. Van Zyl and A. M. Viljoen, "In vitro activity of Aloe extracts against Plasmodium falciparum," South African Journal of Botany, vol. 68, no. 1, pp. 106-110, 2002.

[15] D. K. Patel, R. Kumar, D. Laloo, and S. Hemalatha, "Natural medicines from plant source used for therapy of diabetes mellitus: an overview of its pharmacological aspects," pp. 239250, 2012.

[16] B. Lu, D. Xia, W. Huang, X. Wu, Y. Zhang, and Y. Yao, "Hypolipidemic effect of bamboo shoot oil (P. pubescens) in Sprague-Dawley rats," Journal of Food Science, vol. 75, no. 6, pp. H205-H211, 2010.

[17] N. Taleb-Dida, D. Krouf, and M. Bouchenak, "Globularia alypum aqueous extract decreases hypertriglyceridemia and ameliorates oxidative status of the muscle, kidney, and heart in rats fed a high-fructose diet," Nutrition Research, vol. 31, no. 6, pp. 488-495, 2011.

[18] C. C. Allain, L. S. Poon, and C. S. G. Chan, "Enzymatic determination of total serum cholesterol," Clinical Chemistry, vol. 20, no. 4, pp. 470-475, 1974.

[19] M. Burstein, H. R. Scholnick, and R. Morfin, "Rapid method for the isolation of lipoproteins from human serum by precipitation with polyanions," Journal of Lipid Research, vol. 11, no. 6, pp. 583-595, 1970.

[20] M. W. McGowan, J. D. Artiss, D. R. Strandbergh, and B. Zak, "A peroxidase-coupled method for the colorimetric determination of serum triglycerides," Clinical Chemistry, vol. 29, no. 3, pp. 538-542, 1983.

[21] J. Sedlak and R. H. Lindsay, "Estimation of total, proteinbound, and nonprotein sulfhydryl groups in tissue with Ellman's reagent," Analytical Biochemistry, vol. 25, pp. 192-205, 1968.

[22] H. Ohkawa, N. Ohishi, and K. Yagi, "Assay for lipid peroxides in animal tissues by thiobarbituric acid reaction," Analytical Biochemistry, vol. 95, no. 2, pp. 351-358, 1979.

[23] A. Venugopala Rao and S. Ramakrishnan, "Indirect assessment of hydroxymethylglutaryl CoA reductase (NADPH) activity in liver tissue," Clinical Chemistry, vol. 21, no. 10, pp. 1523-1525, 1975.

[24] A. R. Borate, A. A. Suralkar, S. S. Birje, P. V. Malusare, and P. A. Bangale, "Antihyperlipidemic effect of protocatechuic acid in fructose induced hyperlipidemia in rats," International Journal of Pharma and Bio Sciences, vol. 2, no. 4, pp. 456-460, 2011.

[25] G. L. Kelley, G. Allan, and S. Azhar, "High dietary fructose induces a hepatic stress response resulting in cholesterol and lipid dysregulation," Endocrinology, vol. 145, no. 2, pp. 548-555, 2004.

[26] J. Fu, X. Zhang, K. Liu et al., "Hypolipidemic activity in SpragueDawley rats and constituents of a novel natural vegetable oil from Cornus wilsoniana fruits," Journal of Food Science, vol. 77, pp. 160-169, 2012.

[27] O.-J. Park, D. Cesar, D. Faix, K. Wu, C. H. L. Shackleton, and M. K. Hellerstein, "Mechanisms of fructose-induced hypertriglyceridaemia in the rat. Activation of hepatic pyruvate dehydrogenase through inhibition of pyruvate dehydrogenase kinase," Biochemical Journal, vol. 282, no. 3, pp. 753-757, 1992.

[28] R. Yazdanparast, S. Bahramikia, and A. Ardestani, "Nasturtium officinale reduces oxidative stress and enhances antioxidant capacity in hypercholesterolaemic rats," Chemico-Biological Interactions, vol. 172, no. 3, pp. 176-184, 2008.

[29] P. G. Jain, S. D. Patil, N. G. Haswani, M. V. Girase, and S. J. Surana, "Hypolipidemic activity of Moringa oleifera Lam., Moringaceae, on high fat diet induced hyperlipidemia in albino rats," Brazilian Journal of Pharmacognosy, vol. 20, no. 6, pp. 969973, 2010.

[30] M. Valgimigli, E. Merli, P. Malagutti et al., "Endothelial dysfunction in acute and chronic coronary syndromes: evidence for a pathogenetic role of oxidative stress," Archives of Biochemistry and Biophysics, vol. 420, no. 2, pp. 255-261, 2003.

[31] N. P. Visavadiya and A. V. R. L. Narasimhacharya, "Hypolipidemic and antioxidant activities of Asparagus racemosus in hypercholesteremic rats," Indian Journal of Pharmacology, vol. 37, no. 6, pp. 376-380, 2005.

[32] S. S. Reddy, P. Ramatholisamma, R. Karuna, and D. Saralakumari, "Preventive effect of Tinospora cordifolia against highfructose diet-induced insulin resistance and oxidative stress in male Wistar rats," Food and Chemical Toxicology, vol. 47, no. 9, pp. 2224-2229, 2009.

[33] T. D'Souza, S. Mengi, S. Hassarajani, and S. Chattopadhayay, "Efficacy study of the bioactive fraction (F-3) of Acorus calamus in hyperlipidemia," Indian Journal of Pharmacology, vol. 39, no. 4, pp. 196-200, 2007.

[34] P. O. Bonetti, D. R. Holmes Jr., A. Lerman, and G. W. Barsness, "Enhanced external counterpulsation for ischemic heart disease: what's behind the curtain?" Journal of the American College of Cardiology, vol. 41, no. 11, pp. 1918-1925, 2003.

[35] L. K. Mehta, R. Balaraman, A. H. Amin, P. A. Bafna, and O. D. Gulati, "Effect of fruits of Moringa oleifera on the lipid profile of normal and hypercholesterolaemic rabbits," Journal of Ethnopharmacology, vol. 86, no. 2-3, pp. 191-195, 2003. 

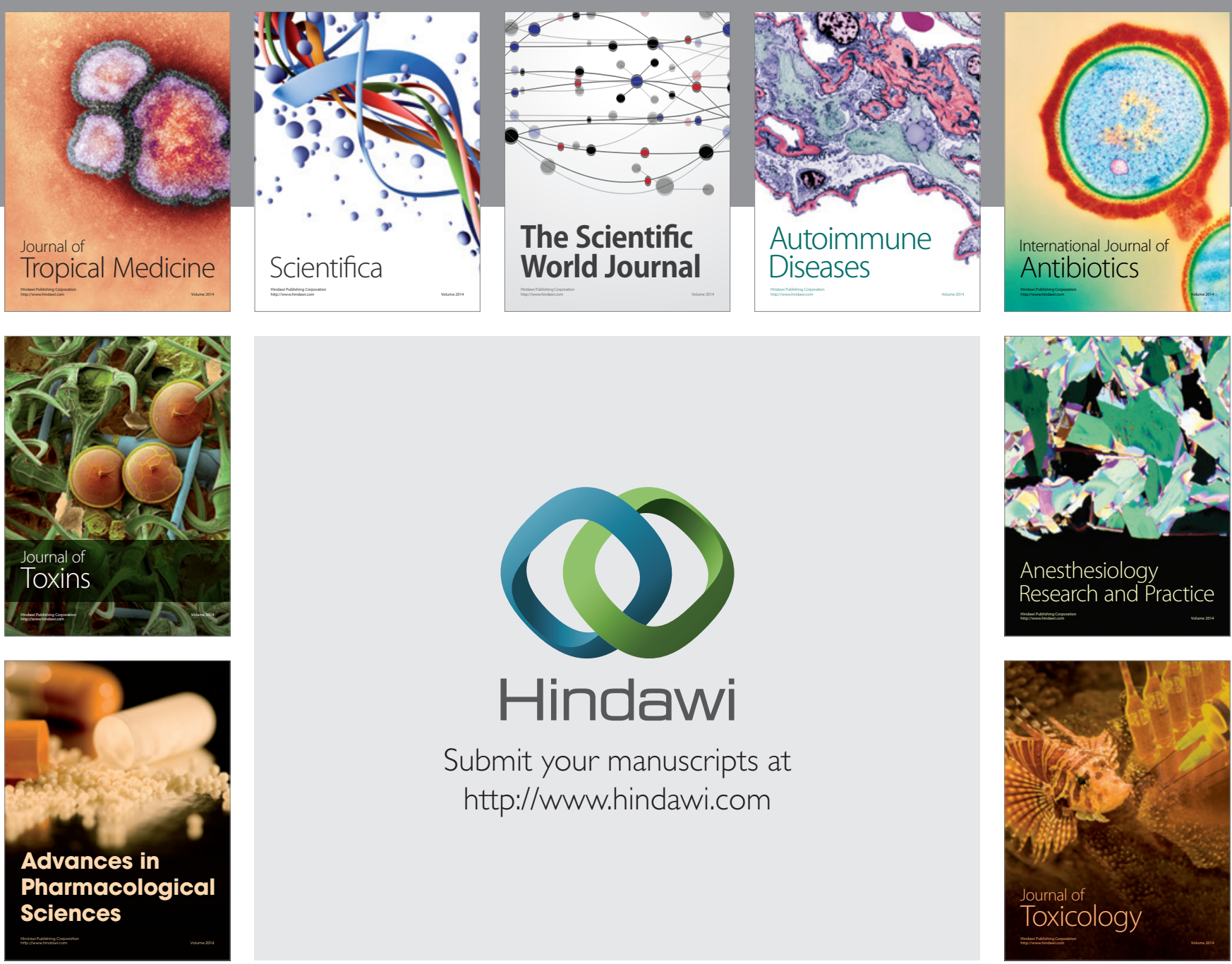

\section{Hindawi}

Submit your manuscripts at

http://www.hindawi.com
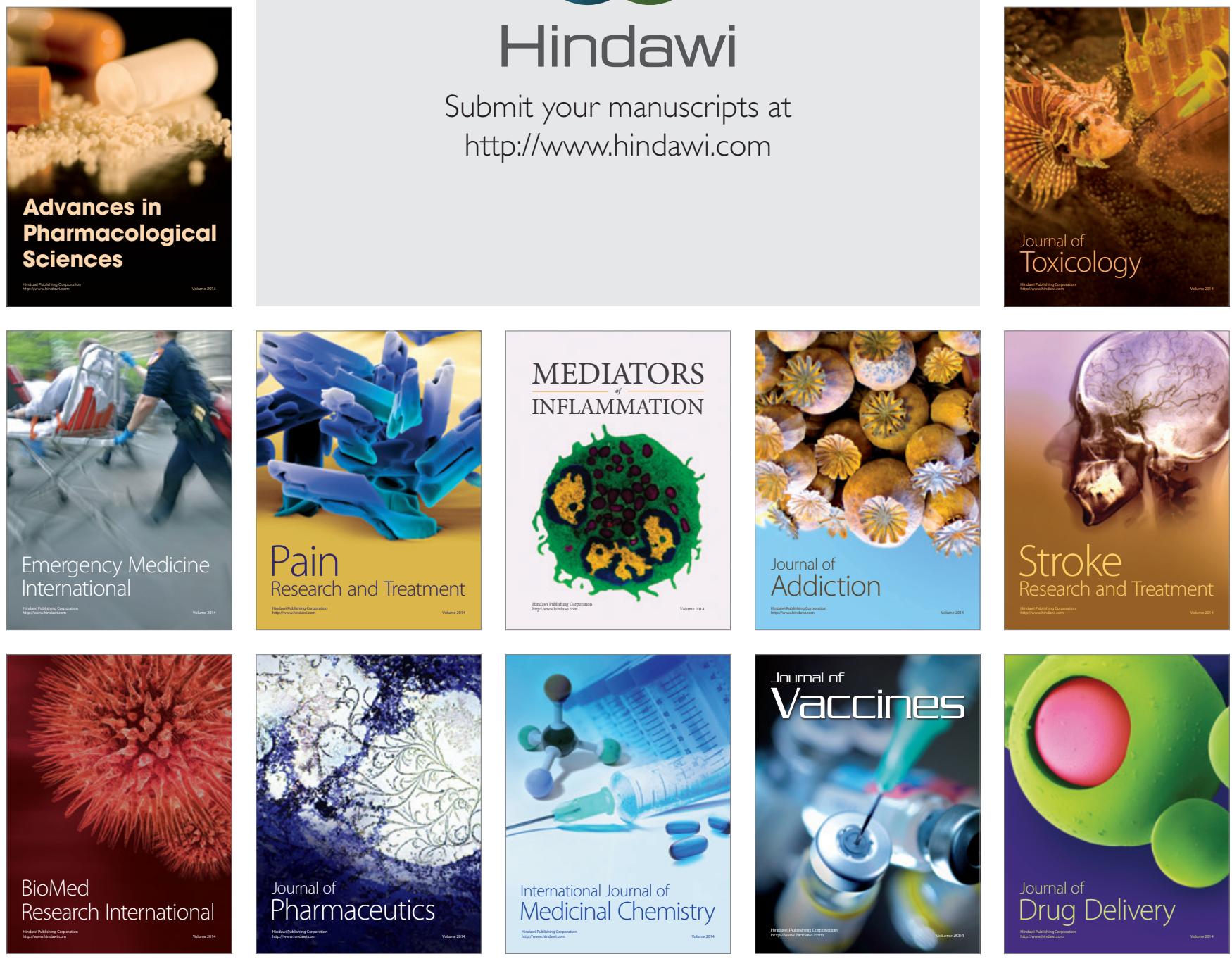